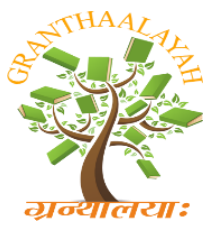

\author{
INTERNATIONAL JOURNAL OF RESEARCH - \\ GRANTHAALAYAH \\ A knowledge Repository
}

\title{
ENVIRONMENTAL PROBLEMS ITS EFFECTS AND APPLICABLE METHODS
}

\section{Nikhat Parveen Ahmed}

Govt. Girls P.G. College Ujjain

\section{HOW PSYCHOLOGY DEAL WITH ENVIRONMENT?}

"Environmental Psychology is field of study that examines the inter relationship between environment and human affect cognition and behaviour" (Bechtel \& chorchman 2002, Gilford 2007)

\section{Environment Types}

Natural / Water / Air / Land / Mountain / Forest / Vegetation

Mane Built - Home / Road / School / College / Market / Industries of etc. General orientation to nature and environment (according Florence Cluson (1953)

- People as subjugated to nature

- People as above nature

- People as part of nature

How Environment affects human body (Change in the environment due to human activities)

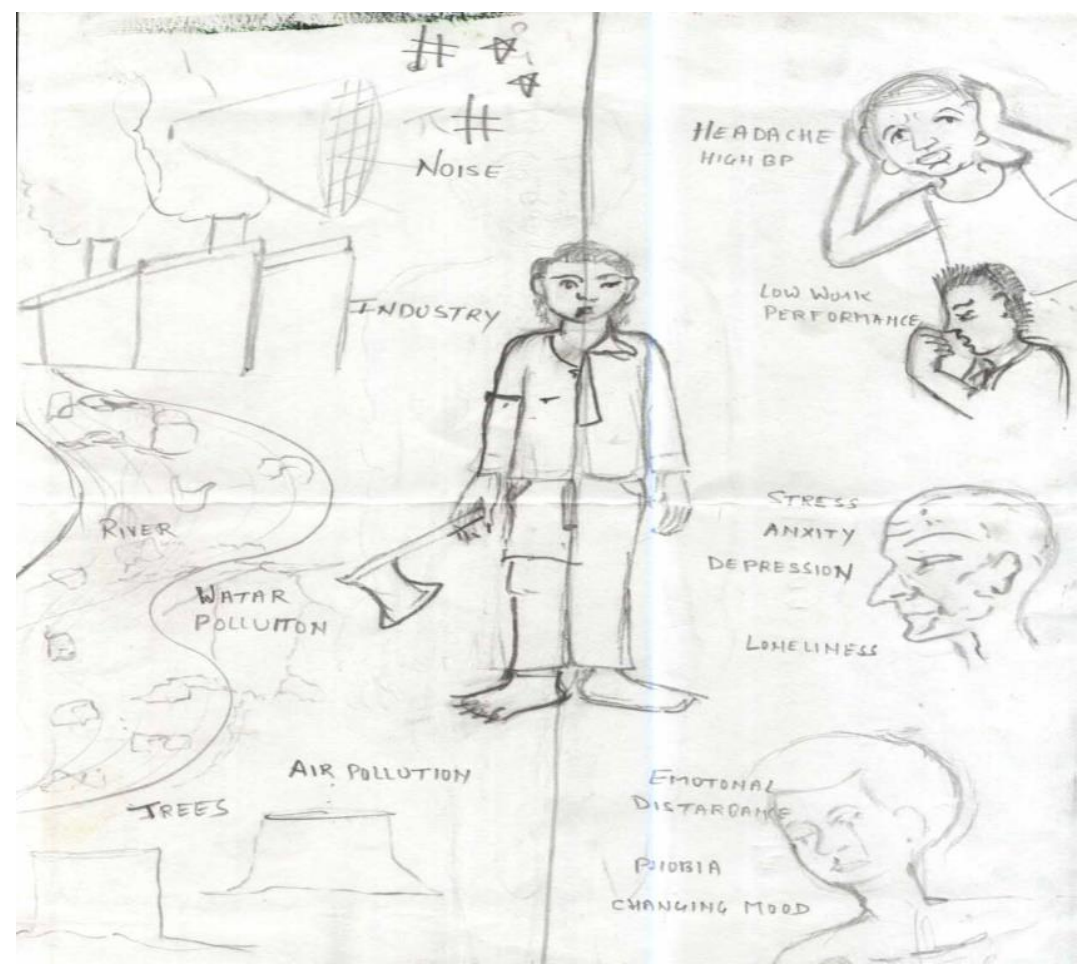


Environmental problems and its effects

\begin{tabular}{|c|c|c|c|c|}
\hline $\begin{array}{c}\text { Environmental } \\
\text { problems }\end{array}$ & Types & Physical & Social & Mental \\
\hline $\begin{array}{l}\text { Natural } \\
\text { Disaster }\end{array}$ & $\begin{array}{l}\text { - Flood } \\
\text { - Tsunami } \\
\text { - Draught } \\
\text { - Snow fall } \\
\text { - Earthquake }\end{array}$ & $\begin{array}{l}\text { - Stunned } \\
\text { - Numb } \\
\text { - Fearlessness } \\
\text { - Irrational }\end{array}$ & $\begin{array}{ll}\text { - } & \text { Separation } \\
\text { - } & \text { Homeless } \\
\text { - } & \text { Scarcity }\end{array}$ & $\begin{array}{l}\text { - Anxiety } \\
\text { - Stress } \\
\text { - Frustration } \\
\text { - Depression } \\
\text { - Phobia } \\
\text { - Loneliness }\end{array}$ \\
\hline $\begin{array}{l}\text { Noise } \\
\text { Unpleasant } \\
\text { sound } \\
\text { (above } 90 \mathrm{db} \text { ) }\end{array}$ & $\begin{array}{l}\text { - Intensity of } \\
\text { noise } \\
\text { - Pitch } \\
\text { - Reflection } \\
\text { - Periodicity }\end{array}$ & $\begin{array}{l}\text { - High livel of } \\
\text { catecholamine } \\
\text { - High blood } \\
\text { pressure } \\
\text { - Digestive } \\
\text { disturbance } \\
\text { - Allergy } \\
\text { - Cardiovascular } \\
\text { disease } \\
\text { - Hearing loss } \\
\text { - Headache }\end{array}$ & 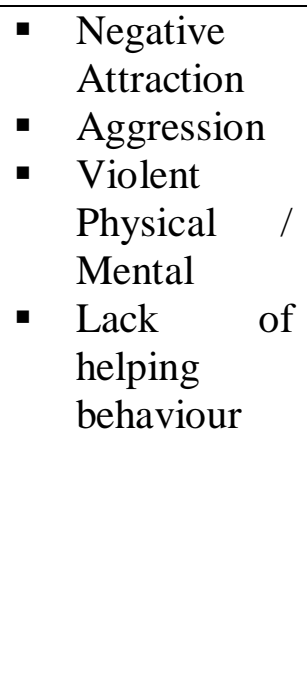 & $\begin{array}{l}\text { - } \text { Lack of } \\
\text { - } \text { Lerceptual control } \\
\text { - } \text { hearned } \\
\text { - Memplessness } \\
\text { - High arousal } \\
\text { level of } \\
\text { - Distraction of } \\
\text { - } \text { attention } \\
\text { - } \text { performance } \\
\text { - } \text { Changing mood } \\
\text { - Anstability } \\
\text { - Anxiety / stress }\end{array}$ \\
\hline $\begin{array}{l}\text { Temperature } \\
\text { and heat } \\
\text { (above } 32^{\circ} \mathrm{C} \text { ) }\end{array}$ & $\begin{array}{l}\text { - Dust storm } \\
\text { - Cyclone } \\
\text { - Tornado }\end{array}$ & $\begin{array}{l}\text { - Effect on } \\
\text { thermo } \\
\text { regulatory } \\
\text { mechanism } \\
\text { - High BP/High } \\
\text { heartbeat, } \\
\text { heart attack } \\
\text { - High skin } \\
\text { conductance } \\
\text { - sweating ros } \\
\text { - used Pelpitation } \\
\text { - Fatigue }\end{array}$ & 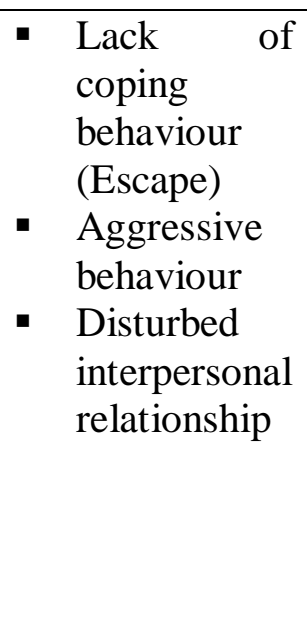 & $\begin{array}{l}\text { - Irritability } \\
\text { - Anxiety } \\
\text { - Low stress } \\
\text { tolerance } \\
\text { - Unhappiness }\end{array}$ \\
\hline Air Pollution & $\begin{array}{l}\text { External } \\
\text { - Ozone } \\
\text { - Sulphur } \\
\text { oxide } \\
\text { - Nitrogen } \\
\text { oxide } \\
\text { - Carbon } \\
\text { mono oxide } \\
\text { - Internal } \\
\end{array}$ & $\begin{array}{l}\text { - Lack of } \\
\text { Oxygen } \\
\text { (Hypoxia) } \\
\text { - Dumb and } \\
\text { Deaf, } \\
\text { Blindness } \\
\text { - Epilepsy } \\
\text { - Headache } \\
\text { - Fatigue }\end{array}$ & $\begin{array}{l}\text { - Low work } \\
\text { performance } \\
\text { - High } \\
\text { reaction time } \\
\text { - Driving } \\
\text { problem } \\
\text { - High rate of } \\
\text { accident }\end{array}$ & 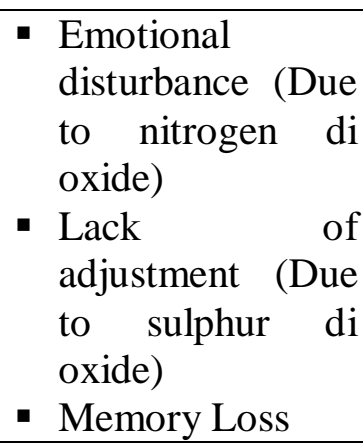 \\
\hline
\end{tabular}




\begin{tabular}{|c|c|c|c|c|}
\hline $\begin{array}{c}\text { Environmental } \\
\text { problems }\end{array}$ & Types & Physical & Social & Mental \\
\hline & $\begin{array}{l}\text { (Industrial/ } \\
\text { homes) } \\
\text { - Sulphur di } \\
\text { oxide } \\
\text { - Nitrogen di } \\
\text { oxide } \\
\text { - Cadmium } \\
\text { - Mercury }\end{array}$ & $\begin{array}{l}\text { - Cancer } \\
\text { - Immunity } \\
\text { - } \text { Air Pollution } \\
\text { syndrome } \\
\text { (APS) }\end{array}$ & $\begin{array}{ll}\text { - } & \text { Lack of } \\
\text { interpersonal } \\
\text { attraction } \\
\text { (due to } \\
\text { ammonia } \\
\text { sulphur di } \\
\text { oxide) } \\
\text { - Increase } \\
\text { aggressive } \\
\text { behaviour } \\
\text { (Due to } \\
\text { ethyl } \\
\text { mrcaptain) }\end{array}$ & $\begin{array}{ll}\text { - } & \text { Mental } \\
& \text { Retardation (MR) }\end{array}$ \\
\hline Water pollution & $\begin{array}{l}\text { - Industrial } \\
\text { waste } \\
\text { - Sewage } \\
\text { waste } \\
\text { - excessive } \\
\text { use of } \\
\text { plastic or } \\
\text { fertilizers } \\
\text { - Carelessness } \\
\text { of people } \\
\text { towords } \\
\text { water } \\
\text { pollution } \\
\text { etc. }\end{array}$ & $\begin{array}{l}\text { - Health hazards } \\
\text { - Reduction in } \\
\text { solar energy } \\
\text { and decreased } \\
\text { rate of } \\
\text { photosynthesis } \\
\text { - Oxygen } \\
\text { deficiency in } \\
\text { water } \\
\text { - Decrease in } \\
\text { fresh water }\end{array}$ & $\begin{array}{l}\text { - Violent } \\
\text { behaviour } \\
\text { - Low quality } \\
\text { of life }\end{array}$ & $\begin{array}{l}\text { - Tension } \\
\text { - Anxiety } \\
\text { - Unhappiness } \\
\text { - Depression }\end{array}$ \\
\hline
\end{tabular}

\section{Applicable methods for the solution of environmental problems}

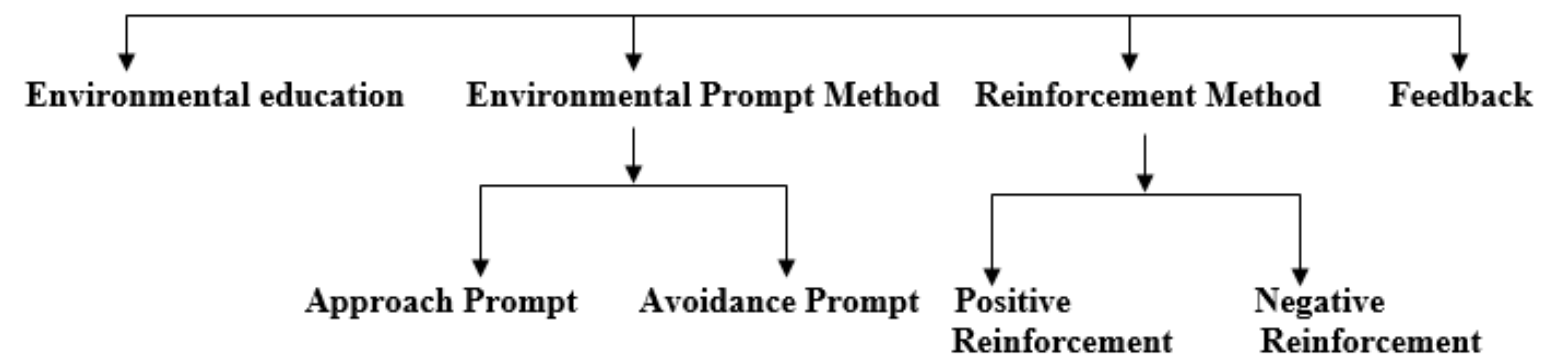

\section{ENVIRONMENTAL EDUCATION}

- Respect for Mother Nature (water / air / land / forest / animals / vegetation.

- Include environment topics in syllabus at school / college / university level. 
- Govt. org / NGOs / semi govt. org / should have awareness programme for environment (organize workshop and seminar).

- Organisation / institution should mark important environment days.

ENVIRONMENTAL PROMPT - Improvement for good behaviour

- Appreciation for appropriate behaviour.

- Punishment and fines for inappropriate behaviour.

- Monetary help /donations.

- Plantations.

- Self-role model.

- Give written information.

- Simple and effective quotations and instruction.

REINFORCEMENT METHOD

- Monetary awards by Govt. (+ve behaviour occur).

- Relaxation in tax benefits (+ve behaviour).

- Makes laws / acts / rules.

- Penalty by increasing tax (-ve).

FEEDBACK - Information about behaviour modification

- Set goals and target.

- Target achieved or not.

- Goal attained or not.

- Feedback result in positive behaviour. 